\title{
Molecular identification and characterization of the platelet ADP receptor targeted by thienopyridine antithrombotic drugs
}

\author{
Carolyn J. Foster, ${ }^{1}$ Dina M. Prosser, ${ }^{2}$ Jacqueline M. Agans, ${ }^{1}$ Ying Zhai, ${ }^{1}$ Michelle D. Smith, ${ }^{1}$ \\ Jean E. Lachowicz, ${ }^{1}$ Fang L. Zhang, ${ }^{3}$ Eric Gustafson, ${ }^{3}$ Frederick J. Monsma, Jr., ${ }^{3}$ \\ Maria T. Wiekowski, ${ }^{2}$ Susan J. Abbondanzo, ${ }^{2}$ Donald N. Cook, ${ }^{2}$ Marvin L. Bayne, ${ }^{3}$ \\ Sergio A. Lira, ${ }^{2}$ and Madhu S. Chintala ${ }^{1}$
}

${ }^{1}$ Department of Central Nervous System and Cardiovascular Pharmacology,
${ }^{2}$ Department of Immunology, and
${ }^{3}$ Department of Human Genome Research, Schering-Plough Research Institute, Kenilworth, New Jersey, USA
Address correspondence to: Carolyn Foster, Schering-Plough Research Institute,
2015 Galloping Hill Road, Kenilworth, New Jersey 07033, USA.
Phone: (908) 740-3244; Fax: (908) 740-3294; E-mail: carolyn.foster@spcorp.com.
Carolyn J. Foster, Dina M. Prosser, and Madhu S. Chintala contributed equally to this work.

Received for publication January 16, 2001, and accepted in revised form May 4, 2001.

\begin{abstract}
ADP plays a critical role in modulating thrombosis and hemostasis. ADP initiates platelet aggregation by simultaneous activation of two G protein-coupled receptors, P2Y1 and P2Y12. Activation of P2Y1 activates phospholipase $\mathrm{C}$ and triggers shape change, while P2Y12 couples to Gi to reduce adenylyl cyclase activity. P2Y12 has been shown to be the target of the thienopyridine drugs, ticlopidine and clopidogrel. Recently, we cloned a human orphan receptor, SP1999, highly expressed in brain and platelets, which responded to ADP and had a pharmacological profile similar to that of P2Y12. To determine whether SP1999 is P2Y12, we generated SP1999-null mice. These mice appear normal, but they exhibit highly prolonged bleeding times, and their platelets aggregate poorly in responses to ADP and display a reduced sensitivity to thrombin and collagen. These platelets retain normal shape change and calcium flux in response to ADP but fail to inhibit adenylyl cyclase. In addition, oral clopidogrel does not inhibit aggregation responses to ADP in these mice. These results demonstrate that SP1999 is indeed the elusive receptor, P2Y12. Identification of the target receptor of the thienopyridine drugs affords us a better understanding of platelet function and provides tools that may lead to the discovery of more effective antithrombotic therapies.
\end{abstract}

J. Clin. Invest. 107:1591-1598 (2001).

\section{Introduction}

ADP is an important mediator of platelet aggregation. Acting through multiple receptors, ADP induces platelet shape change, calcium flux, and inhibition of adenylyl cyclase, leading to aggregation (1-3). ADP is also secreted from activated platelets, thereby amplifying its own effects, as well as those of other activators such as thrombin and collagen $(4,5)$. Pharmacological studies suggest the existence of three ADP receptors on platelets $(2,6)$. P2X1, a Ca ${ }^{+2}$ channel $(7,8)$, and P2Y1 (9-11), a G protein-coupled receptor (GPCR), have been cloned and shown to be present in platelets. P2X1 appears to play a minor role in platelet aggregation (2), while P2Y1 mediates ADP-induced shape change, phospholipase $\mathrm{C}$ activation, and calcium flux $(2,12-14)$. Until recently, the third ADP receptor, a GPCR termed P2Y12 that signals through Gi and inhibits adenylyl cyclase activity $(3,6,9,15,16)$, was not identified at the molecular level. The mechanism of ADP-induced platelet aggregation is complex, since simultaneous activation of P2Y1, coupled to Gq, and P2Y12, coupled to $\mathrm{Gi}$, is necessary to produce a complete aggregation response $(3,9)$. However, if P2Y12 is blocked with a selective inhibitor, the function of the receptor can be replaced by activation of another Gi-coupled receptor, the $\alpha 2 a$-adrenergic receptor (3).

Our understanding of ADP receptor function in platelets has been advanced by studies on P2Y1-null mice $(13,14)$. Platelets from these mice do not aggregate in response to ADP, except partially at very high concentrations of the nucleotide. In addition, ADP does not induce a calcium transient or shape-change response in these platelets. ADP-induced inhibition of adenylyl cyclase is unchanged from wild-type mice, however, showing that $\mathrm{P} 2 \mathrm{Y} 12$ is present. In platelets from $\mathrm{P}_{2} \mathrm{Y}^{-/}$ mice, collagen-induced in vitro aggregation is strongly impaired. In addition, responses to thrombin are reduced at limiting concentrations. $P 2 \mathrm{Y}^{-/-}$mice are also resistant to thromboembolism in vivo induced by intravenous injection of thromboplastin, a thrombin- 
dependent model of thrombosis (17). These results point to a role for P2Y1 in mediating both collagen and thrombin responses in platelets. However, other studies have suggested that $\mathrm{ADP} /$ thrombin interaction requires P2Y12. For example, activation of phosphoinositide 3-kinase (18) and phospholipase D (19) in human platelets by low concentrations of thrombin requires ADP, and these effects are blocked by ARL 66096, a selective inhibitor of P2Y12. In addition, responses to collagen and thrombin are impaired in platelets from an individual with a bleeding disorder that has been linked to a defect in the P2Y12 receptor (20).

From a clinical perspective, P2Y12 is an important receptor, since pharmacological studies demonstrate that this receptor is the target of the widely used antithrombotic drugs, ticlopidine and clopidogrel (16, 21). These drugs have been shown in clinical trials to reduce the incidence of myocardial infarction and stroke in patients with atherosclerotic disease (22).

Recently, we reported the cloning of an orphan GPCR, SP1999, and identified ADP as its ligand (23). The nucleotide sensitivity of SP1999 resembled that of a platelet ADP receptor, and the sequence was distinct from P2Y1. To test the hypothesis that SP1999 was the previously unidentified P2Y12, we generated SP1999null mice. We show here that platelets from SP1999null mice do not respond to ADP with a decrease in cAMP, but they retain the P2Y1-linked functions, calcium flux and shape change. The aggregation response to ADP in SP1999-null platelets is strongly impaired, and bleeding times in SP1999-null mice are prolonged. In addition, clopidogrel has no effect on aggregation in SP1999-null platelets. These results provide strong evidence that SP1999 is indeed P2Y12.

\section{Methods}

Reagents. Thrombin was purchased from Enzyme Research Laboratories (South Bend, Indiana, USA), clopidogrel from Bristol-Myers Squibb Co. (Princeton, New Jersey, USA), fura 2/AM and Pluronic F-127 from Molecular Probes Inc. (Eugene, Oregon, USA), and ${ }^{3} \mathrm{H}-2$-methylthioadenosine diphosphate $\left({ }^{3} \mathrm{H}-2\right.$ MeS-ADP) from Amersham Pharmacia Biotech (Piscataway, New Jersey, USA). The 2-methylthioadenosine monophosphate (2-MeS-AMP) was obtained by custom synthesis from Qventas Inc. (Newark, Delaware, USA). Protease-activated receptor 4-activating peptide (PAR-4 TRAP, Gly-Tyr-Pro-Gly-LysPhe-NH2) was obtained by custom synthesis from AnaSpec Inc. (San Jose, California, USA). Unless otherwise noted, all other reagents were purchased from Sigma Chemical Co. (St. Louis, Missouri, USA).

Generation of SP1999-deficient mice. A bacterial artificial chromosome clone containing the mouse SP1999 (mSP1999) sequence, now identified as P2Y12, was amplified by PCR screening of a mouse 129/SV library (Incyte Genomics Inc., St. Louis, Missouri, USA) using primers derived from the coding sequence of $\mathrm{mSP} 1999$. To construct the targeting vector, $1.2 \mathrm{~kb}$ and 5-kb genomic fragments from the mSP1999 locus were subcloned into the pOSDupDel plasmid (O. Smithies, University of North Carolina, Chapel Hill, North Carolina, USA) such that they flank a neomycin resistance gene (neo). Next, 129/SV mouse embryonic stem (ES) cells were electroporated with the linearized targeting vector and maintained under G418-gancyclovir selection. DNA from drug-resistant colonies was screened for targeted recombination first by a PCR strategy, then by Southern blots of restriction enzyme-digested DNA. ES cell clones containing the predicted DNA structure for the targeted locus were injected into C57BL/6 blastocysts to produce chimeras. To confirm the null phenotype, brain RNA was isolated from $P 2 Y_{12} 2^{-/-}$and $P 2 Y 12^{+/+}$mice, blotted, and probed using P2Y12 cDNA or GAPDH cDNA as a control. Age- and sex-matched $129 / \mathrm{SV} \times \mathrm{C} 57 \mathrm{BL} / 6 \mathrm{~F}_{2}$ control mice from The Jackson Laboratories (Bar Harbor, Maine, USA were initially used as controls. Subsequently, in-house bred $129 / \mathrm{SV} \times \mathrm{C} 57 \mathrm{BL} / 6 \mathrm{~F}_{2}$ control mice derived from the same $129 / \mathrm{SV}$ parent ES cell line as the SP1999-deficient mice were tested. These wild-type mice do not differ genetically from SP1999deficient mice except at the targeted locus. Responses did not differ between the two sets of controls.

Preparation of washed platelets. All animal studies were performed using protocols that were approved by the Schering-Plough Animal Use and Care Committee. Blood was collected from the vena cava of anesthetized mice into syringes containing acid-citrate-dextrose as anticoagulant. Red blood cells were removed by centrifugation at $250 \mathrm{~g}$ for 15 minutes. Platelet-rich plasma (PRP) was recovered, and platelets were pelleted at $1,500 \mathrm{~g}$ for 5 minutes. Except as noted, platelets were resuspended in modified Tyrode's buffer (24) supplemented with $1 \mathrm{mM}$ calcium and $200 \mu \mathrm{g} / \mathrm{ml}$ fibrinogen. Platelet aggregation was performed turbidimetrically, as described by Bednar et al. (24).

Measurement of platelet adenylyl cyclase activity. Production of cyclic AMP in platelets was quantitated using the Adenylyl Cyclase Activation FlashPlate Assay Kit (NEN Life Science Products Inc., Boston, Massachusetts, USA), following the manufacturer's instructions. The $100-\mu \mathrm{l}$ reaction mix contained 50 $\mu \mathrm{l}$ washed platelets $\left(10^{8} / \mathrm{ml}\right)$ in stimulation buffer (NEN-Life Science Products Inc.) containing the phosphodiesterase inhibitor 3-isobutyl-1-methylxanthine. ADP, epinephrine, receptor antagonists, and prostaglandin E1 were dissolved in Dulbecco's PBS (Life Technologies Inc., Rockville, Maryland, USA) supplemented with $0.2 \%$ BSA, $1 \mathrm{~g} / 1$ glucose, and $10 \mathrm{mM} \mathrm{MgCl}$ and were added at the final concentrations indicated in the text and figures. We observed no significant differences in adenylyl cyclase responses between male and female mice.

Intraplatelet calcium-mobilization measurements. Fura2/AM was dissolved at $10 \mathrm{mM}$ in DMSO and diluted 1:1 with $20 \%$ Pluronic F-127 detergent immediately before addition to the platelets. Washed platelets were sus- 


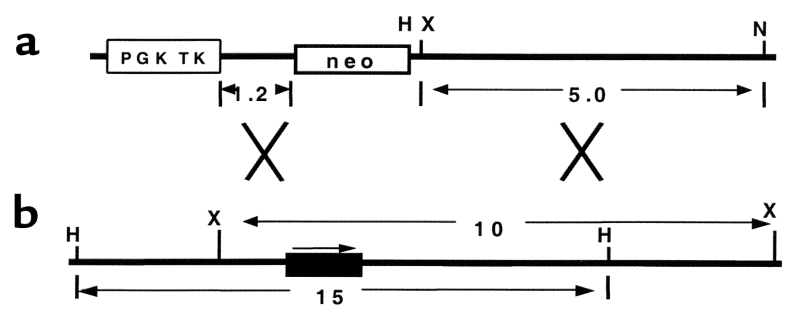

C

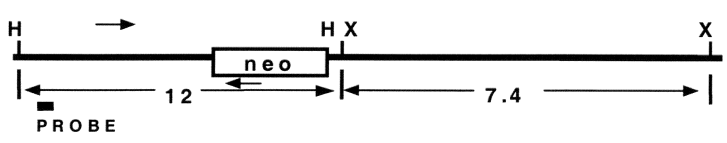

d

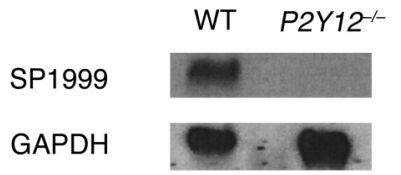

\section{Figure 1}

Targeting the SP1999 (P2Y12) gene. (a) The targeting construct. The neomycin resistance (neo) gene is flanked by $1.2-\mathrm{kb}$ and $5.0-\mathrm{kb}$ DNA fragments from the SP1999 genomic locus. TK is the thymidine kinase gene with the PGK promoter. Restriction sites shown are HindIII $(\mathrm{H})$, Xbal $(\mathrm{X})$, and $\operatorname{Notl}(\mathrm{N})$. Sizes are in kilobases. (b) SP1999 locus. Exonic sequence is shown by a filled rectangle, and an arrow indicates the direction of transcription. (c) Structure of the targeted locus. The sizes of the expected fragments are shown in kilobases. Arrowheads represent the primers used in a PCR to identify targeted ES cell clones. The probe used for Southern blot confirmation of targeted clones is shown. (d) Expression of P2Y12 in adult mouse brain. An SP1999 cDNA probe detects a 2.3-kb species in brain total RNA from wild-type, but not $P 2 Y 12^{-/-}$, mice. GAPDH control probe demonstrates equivalent loading of RNA in each lane.

pended at $10^{8} / \mathrm{ml}$ in modified Tyrode's buffer (24) and loaded with $15 \mu \mathrm{M}$ fura-2/AM dye for 45 minutes at room temperature. Platelets were pelleted and resuspended in Tyrode's buffer (24) at $10^{8} / \mathrm{ml}$. Calcium measurements were performed at $37^{\circ} \mathrm{C}$ with continuous stirring using a Perkin-Elmer LS-50B dual-wavelength fluorometer (Perkin-Elmer Instruments, Norwalk, Connecticut, USA). Excitation wavelengths were 340 and $380 \mathrm{nM}$, and emission was measured at $510 \mathrm{nM}$. Calcium mobilization is expressed as a ratio of the two emission spectra. We observed no significant differences in calcium responses between male and female mice.

Receptor-binding measurements. Intact washed platelets $\left(0.5 \times 10^{8} / \mathrm{ml}\right)$ were incubated at room temperature in the presence of $1 \mathrm{nM}\left[{ }^{3} \mathrm{H}\right]-2-\mathrm{MeS}-\mathrm{ADP}$ and competitive drugs for 2 minutes to 1 hour. The assay was terminated by rapid filtration on Packard GF-B filters (Packard Instrument Co., Meriden, Connecticut, USA), and radioactivity was detected by scintillation counting. Nonspecific binding was determined in the presence of $1 \mu \mathrm{M}$ unlabeled 2-MeS-ADP.

Platelet aggregation. Male mice were anesthetized with a mixture of ketamine and xylazine $(100 \mathrm{mg} / \mathrm{kg}$ ketamine plus $20 \mathrm{mg} / \mathrm{kg}$ xylazine, administered intraperitoneally), and blood was collected into heparinized $(15 \mathrm{U} / \mathrm{ml})$ syringes by cardiac puncture. Blood was centrifuged at $80 \mathrm{~g}$ for 15 minutes at room temperature. The PRP was removed, and the remaining blood was centrifuged at $1,800 \mathrm{~g}$ for 15 minutes at room temperature to obtain platelet-poor plasma $(P)$. Platelet counts were adjusted to 500,000 per microliter with $P$ as diluent. Platelet aggregation in PRP $(250 \mu \mathrm{l})$ was carried out in an optical ChronoLog aggregometer (Chrono-Log Corp., Havertown, Pennsylvania, USA), and $P$ was used as $100 \%$ reference for aggregation. In the clopidogrel experiments, mice were dosed orally with $25 \mathrm{mg} / \mathrm{kg}$ twice a day for 2 days plus a $25 \mathrm{mg} / \mathrm{kg}$ dose 2 hours before obtaining blood for aggregation on day 3 .

Bleeding time. Male mice were anesthetized with a mixture of ketamine and xylazine $(100 \mathrm{mg} / \mathrm{kg}$ ketamine plus $20 \mathrm{mg} / \mathrm{kg}$ xylazine, administered intraperitoneally). The tail was amputated $2 \mathrm{~mm}$ from the tip, and the blood was blotted onto a filter paper every 15 seconds until the bleeding stopped or for up to a maximum of 30 minutes.

Clotting times. Blood was obtained from anesthetized male mice $(100 \mathrm{mg} / \mathrm{kg}$ ketamine plus $20 \mathrm{mg} / \mathrm{kg}$ xylazine, administered intraperitoneally) by cardiac puncture, into citrated syringes. The blood was centrifuged at $1,500 \mathrm{~g}$ for 15 minutes at $4^{\circ} \mathrm{C}$. The activated partial thromboplastin time (aPTT) and prothrombin time (PT), which assess the intrinsic and extrinsic coagulation pathways, respectively, were measured in a COAG-A-MATE machine (Organon Teknika Corp., Durham, North Carolina, USA) with reagents from Organon Teknika Corp. (St. Louis, Missouri, USA) and Sigma Chemical Co.

\section{Results}

P2Y12, a clinically important platelet receptor for ADP, has been difficult to identify at the molecular level (20). During an analysis of human orphan GPCRs, we identified a gene expressed in brain, spinal cord, and platelets (23). This gene coded for a GPCR, SP1999, that responded to nucleotide agonists with the rank order of potency as follows: 2-MeS-ADP $>$ ADP $=$ $\mathrm{ADP} \beta \mathrm{S}>\mathrm{ATP} \gamma \mathrm{S}>2$-Cl-ATP >> $\alpha, \beta$-MeATP (23). Although SP1999 was not closely related in sequence to the P2 family of receptors for extracellular nucleotides, its nucleotide response profile resembled that of P2Y12 $(6,15,25)$. Mouse platelets, similar to human platelets, express a Gi-coupled ADP receptor whose function is blocked by clopidogrel (14). We cloned the mouse SP1999 gene and determined that the predicted amino acid sequence was $86.3 \%$ identical to the human sequence (F. Zhang and D. Prosser, unpublished observations). To investigate the biologic function of SP1999 in platelets, we generated SP1999null mice by targeted gene disruption (Figure 1).

SP1999-null mice were viable, fertile, and displayed no spontaneous bleeding or other overt abnormalities. There was no readily observable phenotype that could indicate the function of SP1999 in the brain. Light 

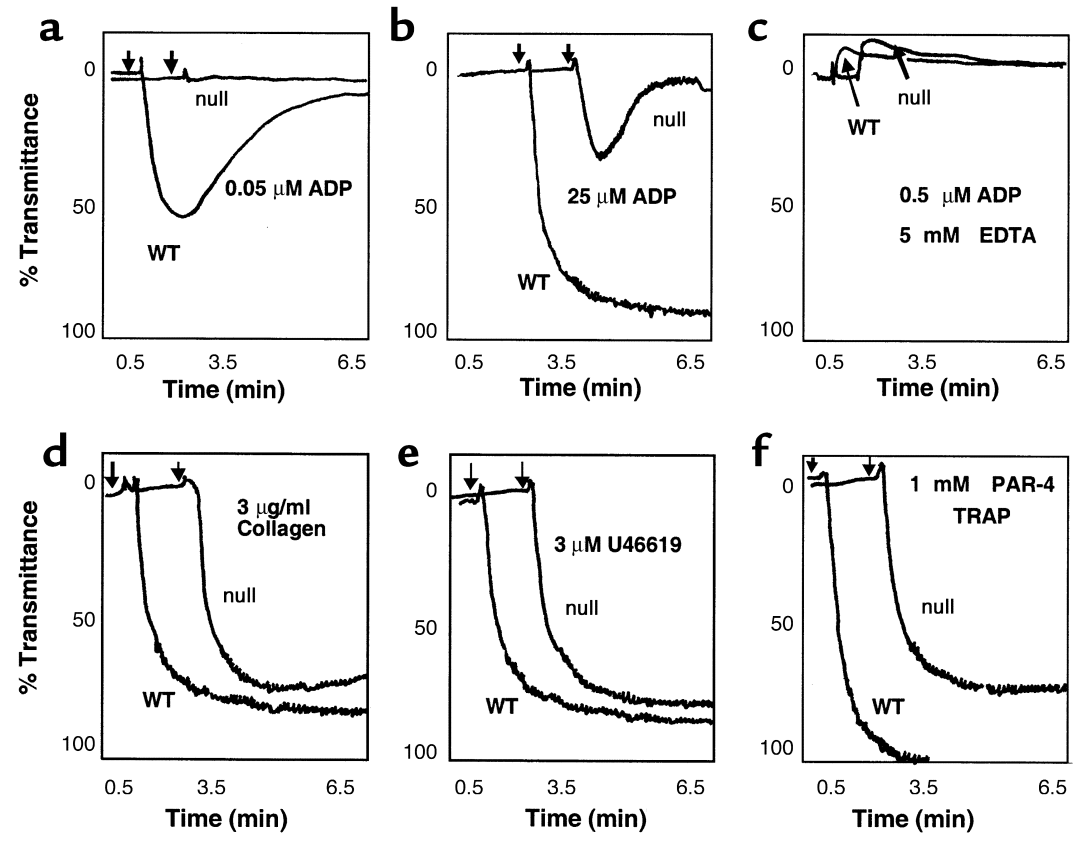

\section{Figure 2}

( $\mathbf{a}$ and $\mathbf{b}$ ) Representative tracings of the platelet aggregation responses to ADP in PRP from SP1999-null and wild-type (WT) mice in vitro. Aggregation responses to ADP are significantly impaired in the SP1999-null mice. Doses up to 500-fold (25 $\mu \mathrm{M})$ (b) higher than the $\mathrm{EC}_{50}(0.05 \mu \mathrm{M}$ in a) in the WT mice elicit only a partial (20-30\%) aggregation response that is rapidly reversible versus the complete and irreversible aggregation seen in the WT mice; $n=4-6$ per dose group. (c) Representative tracings of the shape-change response seen in the presence of $5 \mathrm{mM}$ EDTA to block aggregation. (d-f) Representative tracings of the platelet aggregation responses to other activating agents. (d) Collagen $(3 \mu \mathrm{g} / \mathrm{ml}) ;(\mathbf{e}) \cup 46619(3 \mu \mathrm{M})$, a thromboxane mimetic; (f) PAR-4 TRAP, Gly-Tyr-Pro-Gly-Gln-Val (1 mM), a selective ligand for PAR-4. Platelets from the SP1999-null mice exhibit a complete and irreversible aggregation in response to platelet agonists other than ADP; $n=3-4$ per dose group.

microscopic examination of histologic tissue sections did not reveal abnormalities in any major organ. Blood platelet numbers did not differ significantly between wild-type $\left(87.3 \pm 8.4 \times 10^{4}\right.$ per microliter; $\left.n=4\right)$ and SP1999-null mice $\left(97.2 \pm 12.1 \times 10^{4}\right.$ per microliter; $n=4)$, and other hematologic parameters were also normal in SP1999-null mice (data not shown). These data demonstrate that SP1999 is not required for the development of hematologic lineages, including platelets. When platelets are exposed to ADP, collagen, thrombin, and other activators in injured blood vessels, they become activated and aggregate. To determine the role of SP1999 in platelet function, we first isolated PRP and measured aggregation in vitro. Platelets from wild-type mice underwent a complete, irreversible, and dosedependent aggregation in response to ADP, with an $\mathrm{EC}_{50}$ of $0.05 \mu \mathrm{M}$ (Figure 2a). In contrast, $0.05 \mu \mathrm{M}$ ADP had no measurable effect on platelets of SP1999-null mice. Even at concentrations of ADP 500-fold higher than the $\mathrm{EC}_{50}$ for wild-type mouse platelets, only modest (20-30\%) aggregation responses were seen, and they were rapidly reversible (Figure $2 b$ ). However, SP1999null platelets retained the transient upward deflection of the light transmittance curve that is a measure of the shape-change response to ADP (Figure 2c). Therefore, SP1999 is essential for a maximal and irreversible aggregation response to ADP, but not for shape change.
When adequate concentrations of other platelet agonists such as collagen $(3 \mu \mathrm{g} / \mathrm{ml})$, the thromboxane mimetic U46619 $(3 \mu \mathrm{M})$, or the mouse thrombin receptor tethered ligand peptide, PAR-4 TRAP $(1 \mathrm{mM})$ were tested, platelets from SP1999-null mice exhibited complete and irreversible aggregation responses (Figure 2, d-f). This suggests that the loss of function in SP1999-null mice is specific for ADP and not part of a general defect in platelet aggregation. However, since secreted ADP is known to promote aggregation in response to other agonists such as thrombin and collagen $(4,5)$, we examined aggregation at limiting agonist concentrations as well. To study the effects of thrombin in the absence of plasma coagulation factors, we turned to a washed platelet aggregation assay. As shown in Figure 3, the concentration response curves for both thrombin and collagen were shifted to higher concentrations in the SP1999-null mice as compared with wild-type mice. The $\mathrm{EC}_{50}$ values for thrombin-induced aggregation were 0.07 and $0.2 \mathrm{nM}$ in washed wild-type and SP1999-null platelets, respectively (mean, $n=2$ ). When wild-type platelets were pretreated with the P2Y12selective antagonist, 2-MeS-AMP (12), the $\mathrm{EC}_{50}$ for thrombin shifted to $0.2 \mathrm{nM}$, the value observed in SP1999-null platelets (data not shown). For collagen, the shift in $\mathrm{EC}_{50}$ values in SP1999-null platelets was slightly larger than for thrombin, 0.4 and $4.2 \mu \mathrm{g} / \mathrm{ml}$ for wild-type and SP1999null platelets, respectively (mean, $n=2$ ). The modest shifts 

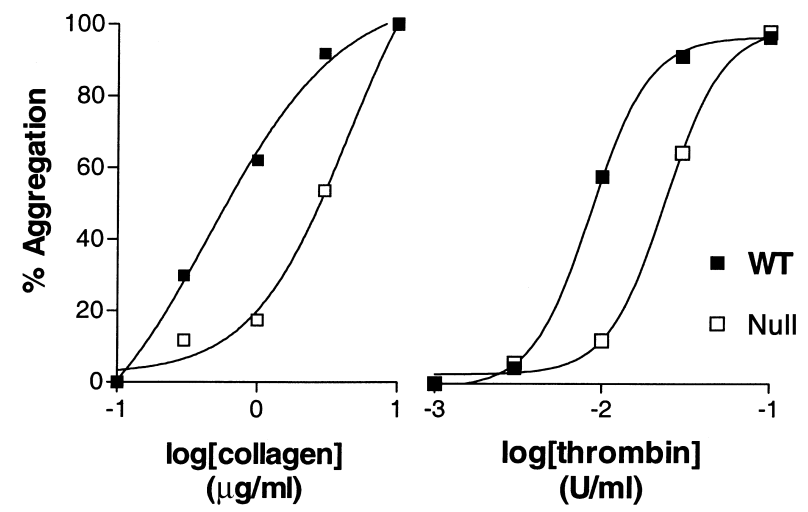

Figure 3

Concentration response curves for platelet aggregation. Washed platelet aggregation was tested using the Bednar technique (24). The extent of aggregation was determined after 2 minutes. Each data point represents the average of three determinations. The plots were derived from a single typical experiment that was repeated two to three times with similar results.

in $\mathrm{EC}_{50}$ values seen with agents other than ADP indicate that SP1999 participates in the response to these agents, probably as a result of release of platelet ADP stores.

It has been demonstrated in human platelets that ADP-induced platelet aggregation requires simultaneous activation of signaling events mediated by $\mathrm{Gi}$ and $\mathrm{Gq}$ (3). If the Gi pathway is blocked by the specific P2Y12 antagonist, ARL 66096, ADP fails to induce aggregation by itself, but aggregation can be restored if $\mathrm{Gi}$ signaling is stimulated by activation of $\alpha-2 a$-adrenergic receptors. We performed a similar experiment using washed SP1999-null platelets (Figure 4). Washed wild-type platelets (Figure 4a) did not aggregate in response to 1 $\mu \mathrm{M}$ epinephrine, but they did aggregate in response to 2 $\mu \mathrm{M}$ ADP. Platelets from SP1999-null mice also failed to aggregate in response to epinephrine (Figure 4b), although they were able to aggregate completely in response to $0.3 \mathrm{U} / \mathrm{ml}$ thrombin. In contrast to the wildtype platelets, SP1999-null platelets failed to aggregate in response to $2 \mu \mathrm{M} \mathrm{ADP}$ (Figure $4 \mathrm{c}$ ), but when ADP and epinephrine were added together, the aggregation was equal to the response of wild-type platelets to ADP alone. These results suggest that SP1999, similar to P2Y12, signals through the Gi pathway.

Hemostasis involves both platelet aggregation and blood coagulation. To assess the relative importance of SP1999 in hemostasis, bleeding times were determined in mice after tail-tip amputation (Figure 5). The mean bleeding time in wild-type mice was 3.5 minutes $(n=7)$, whereas all SP1999-null mice continued to bleed for more than 30 minutes $(n=7)$, at which point the experiments were terminated. To determine whether a defect in coagulation contributed to this large increase in bleeding time, we assessed the aPTT and PT as indices of the intrinsic and extrinsic coagulation pathways, respectively. Neither aPTT nor PT was altered in the SP1999-null mice (Figure 5). These results demonstrate that SP1999 has an important function in hemostasis that is mediated through platelet aggregation, not coagulation.

The nucleotide response profile of SP1999 (23) and the reduced aggregation response, coupled with retention of shape change in SP1999-null platelets, suggest that SP1999 might be the Gi-coupled platelet ADP receptor, $\mathrm{P} 2 \mathrm{Y} 12$. We performed a series of experiments to test this hypothesis. Pharmacological studies have shown that $\mathrm{P} 2 \mathrm{Y} 12$, but not P2Y1, mediates ADP-induced inhibition of adenylyl cyclase activity in platelets $(3,6,9,15,16)$. Therefore, we treated platelets with $1 \mu \mathrm{M}$ prostaglandin E1 (PGE1) to activate adenylyl cyclase and examined the effect of ADP. In wild-type platelets, ADP inhibited PGE1-stimulated cAMP levels in a concentrationdependent fashion (Figure 6a), an effect that was blocked by the selective P2Y12 receptor antagonist, 2-MeS-AMP (Figure 6a). By contrast, platelets of SP1999-null mice showed no measurable decrease in PGE1-stimulated cAMP levels at up to $10 \mu \mathrm{M}$ ADP. To determine whether the Gi-mediated adenylyl cyclase inhibition mechanism was intact in SP1999-null mice, we tested the ability of the $\alpha$-adrenergic receptor agonist, epinephrine, to inhibit adenylyl cyclase. Epinephrine inhibited adenylyl cyclase equally in wild-type and SP1999-null mice (Figure 6a), demonstrating that the Gi signaling pathway is functional in SP1999-null platelets and that the SP1999mediated inhibition of adenylyl cyclase is ADP specific.

In addition to modulation of adenylyl cyclase activity, ADP also induces intracellular calcium mobilization in platelets. This effect, which requires P2Y1 (25), is necessary for maximal platelet aggregation. To determine whether deletion of SP1999 affects calcium flux, we measured intraplatelet calcium levels fluoro-

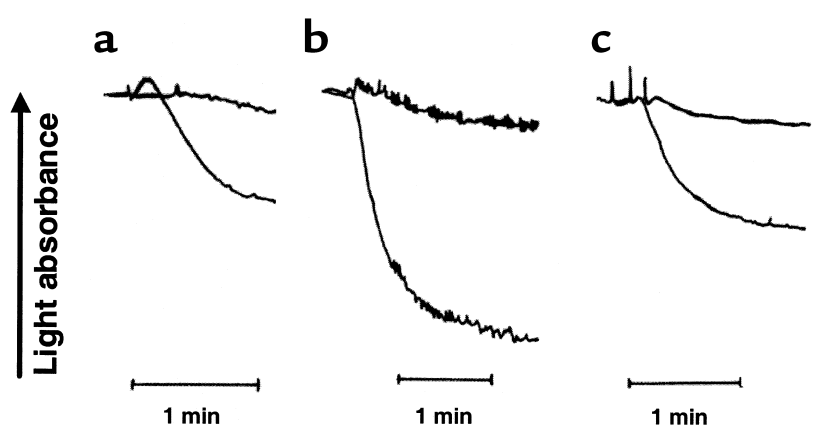

\section{Figure 4}

Representative aggregometer tracings of washed platelet aggregation experiments showing the interactions of ADP and epinephrine. (a) Wild-type platelets were treated with $1 \mu \mathrm{M}$ epinephrine (top curve) or $2 \mu \mathrm{M}$ ADP (bottom curve). (b) SP1999-null platelets were treated with $1 \mu \mathrm{M}$ epinephrine (top curve) or $0.3 \mathrm{U} / \mathrm{ml}$ thrombin (bottom curve). (c) SP1999-null platelets were treated with $2 \mu \mathrm{M}$ ADP (top curve) or a combination of $2 \mu \mathrm{M}$ ADP and $1 \mu \mathrm{M}$ epinephrine (bottom curve). Platelets from four mice were pooled and resuspended in modified Tyrode's buffer supplemented with $1 \mathrm{mM}$ aspirin and $200 \mu \mathrm{g} / \mathrm{ml}$ fibrinogen for each experiment. Aggregation was determined using a Chrono-Log aggregometer. Each experiment was performed three times with similar results. 

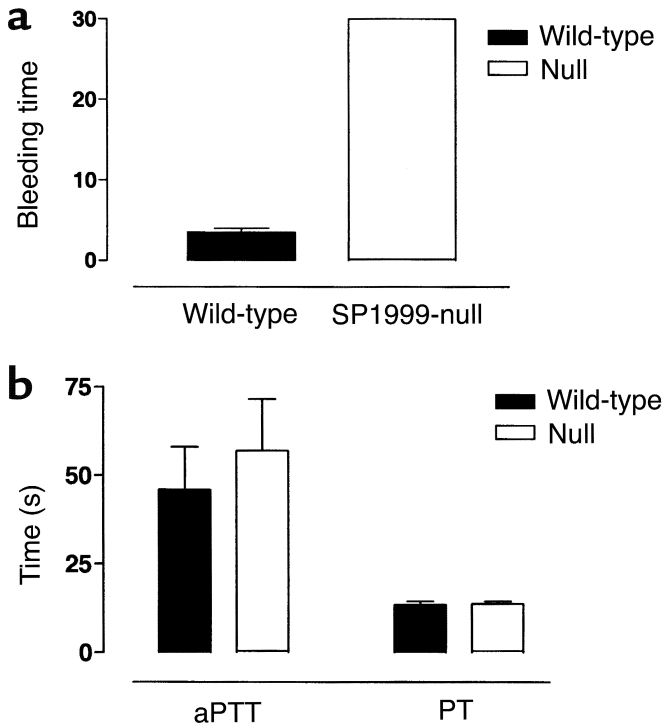

Figure 5

Hemostasis and thrombosis. (a) Bleeding time in SP1999-null $(n=7)$ and wild-type mice $(n=10)$. (b) Clotting times. The aPTT and PT, which are indices of the intrinsic and extrinsic coagulation pathways, were unchanged in the SP1999-null $(n=4)$ and wild-type mice $(n=5)$.

metrically using the calcium-sensitive dye, fura- 2 . Both wild-type and SP1999-null platelets produced robust spikes in intraplatelet calcium in response to ADP challenge (Figure 6b), demonstrating that SP1999 is not required for this activity.

We used selective antagonists of ADP receptor subtypes in a binding assay to confirm the identity of SP1999. Using washed mouse platelets, we demonstrated that there was specific binding of $\left[{ }^{3} \mathrm{H}\right]-2-\mathrm{MeS}-\mathrm{ADP}$ in wild-type platelets that could be displaced by 2 -MeSAMP, but not by the selective P2Y1 antagonist, 3'5-ADP (A3P5P; Figure 6c). No specific binding was measured in SP1999-null platelets. These results support the identification of SP1999 as P2Y12. In these studies, no significant binding to P2Y1 was detected, even though 2-MeS$\mathrm{ADP}$ is a high-affinity ligand for P2Y1 that has been shown to bind to this receptor on washed rat platelets (16). The calcium studies reported above show that functional P2Y1 receptors exist on both wild-type and SP1999-null platelets, but our inability to detect binding may be due to a low receptor number $(1,9)$. It has been reported that platelet $\mathrm{P} 2 \mathrm{Y} 1$ receptors downregulate after a prolonged incubation with ADP, while $\mathrm{P} 2 \mathrm{Y} 12$ receptors remain stable (26). The results shown in Figure 4 were obtained after a 1-hour incubation. In a detailed time-course study (data not shown), no P2Y1 binding was observed after incubation times of 2, 5, 15, 30, or 60 minutes. Our results are similar to those reported for human platelets by Jantzen et al. (12).

The results presented here contrast with those described for mice lacking the other platelet ADP-binding GPCR, P2Y1. In the P2Y1-null mice, the ADP-mediated inhibition of adenylyl cyclase activity is normal, but calcium flux is impaired $(13,14)$. Both SP1999-null and P2Y1-null platelets exhibit marked impairment of ADP-induced aggregation, but SP1999-null platelets retain shape change, while the shape change response is absent from P2Y1-null platelets. Thus, platelets from SP1999-null mice display the properties expected for mice that express P2Y1, but lack P2Y12.

Clopidogrel is a drug that is currently used for the treatment of thrombosis (22). After oral dosing, clopidogrel is metabolized to produce an active species that

\section{Figure 6}

In vitro characterization of washed platelets prepared from wild-type and SP1999-null mice. (a) ADP-induced inhibition of adenylyl cyclase. Wild-type platelets, filled bars; SP1999-null platelets, open bars. PGE1-induced cAMP production is inhibited by ADP in a concentration-dependent manner in platelets from wild-type, but not SP1999-null, mice. In wild-type platelets, inhibition of cAMP production by $10 \mu \mathrm{M}$ ADP is blocked by the selective P2Y12 antagonist, 2-MeS-AMP (or 2$\mathrm{MeS} ; 100 \mu \mathrm{M})$. Epinephrine (EPI) at 1 or $10 \mu \mathrm{M}$ inhibits CAMP production in platelets from both wild-type and SP1999-null mice. Results are mean plus or minus SD of triplicate determinations. Maximum cAMP production was $250 \mathrm{pmol} / 10^{8}$ platelets. (b) Representative tracings of ADP-induced calcium mobilization in platelets from wild-type and SP1999-null mice. Platelets from two mice were pooled for each measurement. Intracellular calcium mobilization was initiated by addition of $50 \mu \mathrm{M}$ ADP at the time indicated on the graph. (c) $\left[{ }^{3} \mathrm{H}\right]-$ 2MeS-ADP binding to washed platelets. Wild-type platelets, filled bars; SP1999-null platelets, open bars. Platelets from wild-type mice, but not SP1999-null mice, bound [ $\left.{ }^{3} \mathrm{H}\right]$-2MeSADP specifically, and the selective P2Y12 antagonist, 2-MeSAMP $(100 \mu \mathrm{M})$, but not the selective P2Y1 antagonist, A3P5P $(100 \mu \mathrm{M})$, displaced this binding. Bars represent the average plus or minus SD of triplicate determinations.

a

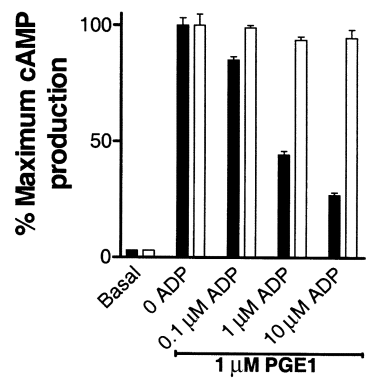

b

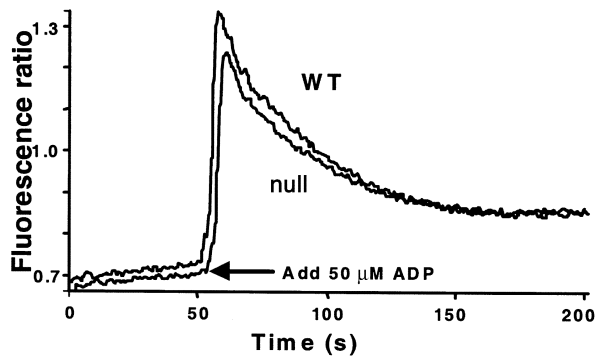

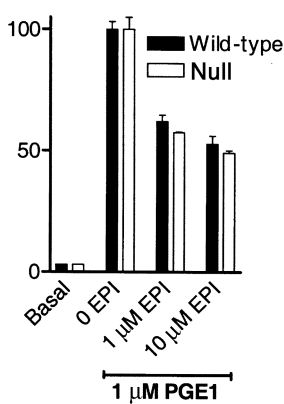

C

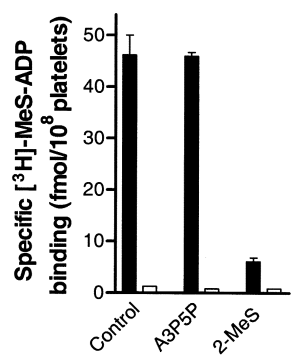



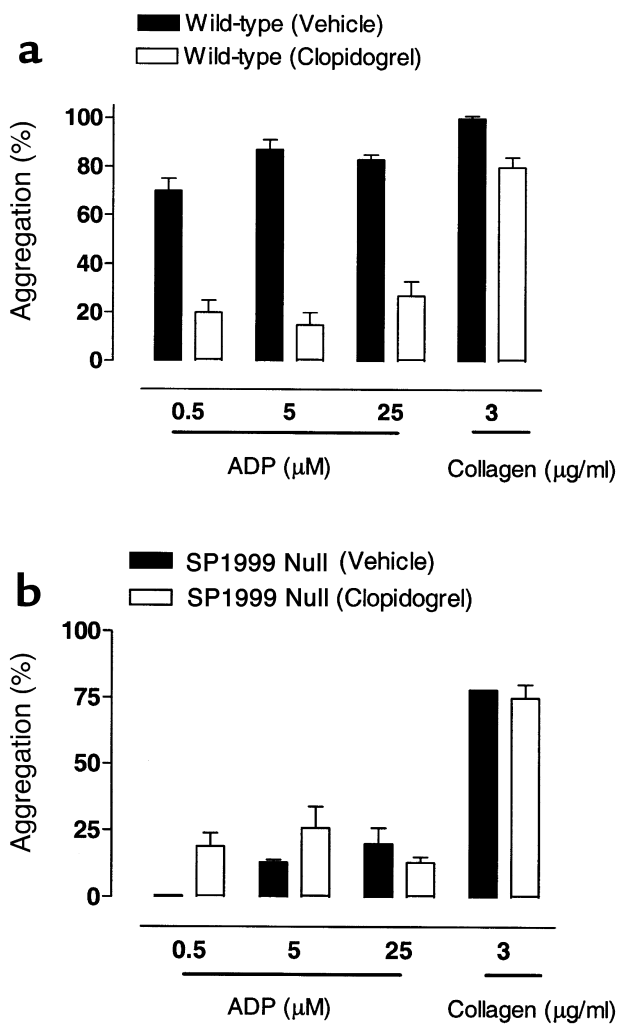

Figure 7

(a) Effects of vehicle or clopidogrel ( $25 \mathrm{mg} / \mathrm{kg}$, twice a day for 2 days plus $25 \mathrm{mg} / \mathrm{kg} 2$ hours before blood withdrawal on day 3 ) on ADPand collagen-induced aggregation in wild-type mice. ADP-induced aggregation was markedly inhibited in the clopidogrel-treated group ( $25 \%$ vs. $85 \%$ in the drug- and vehicle-treated groups, respectively). Collagen-induced aggregation was modestly inhibited by the clopidogrel treatment; $n=4-6$ per group. (b) Effects of vehicle or clopidogrel ( $25 \mathrm{mg} / \mathrm{kg}$, twice a day for 2 days plus $25 \mathrm{mg} / \mathrm{kg} 2$ hours before blood withdrawal on day 3 ) on ADP- and collagen-induced aggregation in SP1999-null mice. ADP-induced aggregation in the drug- and vehicletreated groups were similar (20\%), suggesting that platelets from SP1999-null mice were insensitive to clopidogrel. $n=4-6$ per group.

targets a platelet ADP receptor. The receptor target of the clopidogrel metabolite is presumed to be P2Y12 (16, 21). We therefore evaluated the effects of clopidogrel treatment on ADP-induced aggregation in wild-type and SP1999-null mice (Figure 7). Clopidogrel markedly inhibited the aggregation responses to ADP in wildtype mice ( $85 \%$ vs. $25 \%$ maximal aggregation in the vehicle- and drug-treated groups, respectively). In contrast, clopidogrel treatment had no effect on platelets from SP1999-null mice, where approximately $20 \%$ aggregation was observed in both treated and untreated platelets in response to $5 \mu \mathrm{M}$ ADP. The residual aggregation response to ADP in platelets from both wild-type and SP1999-null mice that had been treated with clopidogrel was abolished by the addition of the P2Y1-selective antagonist, A3P5P (50 $\mu \mathrm{M}$, data not shown). These results show that blocking both P2Y1 and P2Y12 completely inhibits ADP-induced platelet aggregation.

\section{Discussion}

The data presented here provide compelling evidence that SP1999 is the previously unidentified platelet ADP-binding receptor, P2Y12. While this work was in progress, Hollopeter et al. (27) reported cloning of a receptor with a sequence identical to SP 1999 using a mouse oocyte expression cloning strategy. They also identified this receptor to be the platelet ADP receptor, and, following the accepted convention for naming nucleotide receptors, termed it P2Y12 (27). With the identification of P2Y12, the catalogue of pharmacologically defined ADP receptors in platelets appears to be complete. P2Y1 and P2Y12 receptors together are sufficient to account for ADP's known effects on platelets. However, it is not possible to rule out the existence of another $\mathrm{G}$ protein-coupled $\mathrm{ADP}$ receptor, assuming that this receptor could not produce an ADP response independently. P2X1 is a minor player (3), or, as recently reported, it may in fact be a receptor for ATP rather than ADP (28). Previously published studies with P2Y1-deficient mice demonstrated that P2Y1 accounts for the ability of ADP to induce shape change and the large intraplatelet calcium mobilization that precedes aggregation $(13,14)$. Our studies demonstrate that P2Y12 is required to couple ADP to inhibition of adenylyl cyclase in mouse platelets. P2Y12 and P2Y1 appear to be independently regulated, since each retains full function in the absence of the other. We have shown here that P2Y1mediated calcium flux is unchanged in $P 2 \mathrm{Y}^{-/-}$mice, demonstrating that $\mathrm{P} 2 \mathrm{Y} 12$ does not participate in the calcium response, and that there is no apparent compensatory increase of $\mathrm{P} 2 \mathrm{Y} 1$ function in the knockout mice. Similarly, the P2Y12 cyclase inhibition response was unaltered in $\mathrm{P} \mathrm{Y1}^{-/-}$mice $(13,14)$. Although the receptors signal independently, their signaling pathways work synergistically to produce aggregation. Our studies demonstrate that, even at ADP concentrations well above physiological levels, activation of P2Y1 alone produces only a small, transient platelet aggregation response. The aggregation response can be restored toward normal by stimulation of the Gi pathway by addition of epinephrine. The physiological importance of this loss of sustained aggregation is shown by the extensive prolongation of bleeding time that we observed in $P 2 \mathrm{Y}^{-/-}$mice. Similar studies with $P 2 \mathrm{Y1}^{-/-}$mice showed only partial aggregation at very high ADP concentrations and modestly prolonged bleeding times $(13,14)$.

These observations contrast with the effects of another important platelet activator, thrombin. Human platelets express two G protein-coupled thrombin receptors, PAR-1 and PAR-4, but, unlike the ADP receptors, activation of either PAR-1 or PAR-4 independently produces complete platelet aggregation (29). Undoubtedly, the existence of two independent but interactive ADP-receptor systems on platelets, coupled with the release of ADP from activated platelets by other agonists, provides a pow- 
erful mechanism for modulating the essential role of platelets in hemostasis. The $P 2 Y_{12}^{-/-}$mice reported here will be of great value in studies aimed at understanding that mechanism. Clopidogrel is an effective antithrombotic drug that provides significant protection against heart attack and stroke (22). However, it is a prodrug that must be metabolized to an active species that binds covalently to the receptor. As a consequence of this mechanism, the drug must be given for several days before the maximum clinical effect is observed, and elimination of the drug is also slow. Identification of the structure of the target receptor for clopidogrel provides a tool that may lead to discovery of antithrombotic agents with improved efficacy and pharmacokinetic profiles.

\section{Acknowledgments}

The authors thank Maureen Maguire, Brian Wilburn, Shijun Yang, Channa Young, and Petronia Zalamea for experimental assistance; Oliver Smithies, University of North Carolina at Chapel Hill, for his gift of the pOSDupDel plasmid; and Catherine Strader and Michael Graziano for their help and support.

1. Mills, D.C.B. 1996. ADP receptors on platelets. Thromb. Haemost. 76:835-856.

2. Daniel, J.L., et al. 1998. Molecular basis for ADP-induced platelet activation. I. Evidence for three distinct ADP receptors on human platelets. J. Biol. Chem. 273:2024-2029.

3. Jin, J., and Kunapuli, S.P. 1998. Coactivation of two different G proteincoupled receptors is essential for ADP-induced platelet aggregation. Proc. Nat. Acad. Sci. USA. 95:8070-8074.

4. Gachet, C., et al. 1997. Activation of ADP receptors and platelet function. Thromb. Haemost. 78:271-275.

5. Cattaneo, M., et al. 1990. Released adenosine diphosphate stabilizes thrombin-induced human platelet aggregates. Blood. 75:1081-1086.

6. Geiger, J., Honig-Liedl, P., Schanzenbacher, P., and Walter, U. 1998. Ligand specificity and ticlopidine effects distinguish three human platelet ADP receptors. Eur. J. Pharmacol. 351:235-246.

7. Vial, C., Hechler, B., Leon, C., Cazenave, J.-P., and Gachet, C. 1997. Presence of $\mathrm{P} 2 \mathrm{X} 1$ purinoceptors in human platelets and megakaryoblastic cell lines. Thromb. Haemost. 78:1500-1504.

8. Sun, B., Li, J., Okahara, K., and Kambayashi, J. 1998. P2X1 purinoceptor in human platelets. Molecular cloning and functional characterization after heterologous expression. J. Biol. Chem. 273:11544-11547.

9. Savi, P., et al. 1998. Role of P2Y1 purinoceptor in ADP-induced platelet activation. FEBS Lett. 422:291-295.

10. Leon, C., et al. 1997. The P2Y1 receptor is an ADP receptor antagonized by ATP and expressed in platelets and megakaryoblastic cells. FEBS Lett. 403:26-30.
11. Leon, C., Vial, C., Cazenave, J.-P., and Gachet, C. 1996. Cloning and sequencing of a human cDNA encoding endothelial P2Y1 purinoceptor. Gene. 171:295-297.

12. Jantzen, H.-M., et al. 1999. Evidence for two distinct G-protein-coupled ADP receptors mediating platelet activation. Thromb. Haemost. 81:111-117.

13. Fabre, J.-E., et al. 1999. Decreased platelet aggregation, increased bleeding time, and resistance to thromboembolism in P2Y1-deficient mice. Nat. Med. 5:1199-1202.

14. Leon, C., et al. 1999. Defective platelet aggregation and increased resistance to thrombosis in purinergic P2Y1 receptor-null mice. J. Clin. Invest. 104:1731-1737.

15. Jin, J., Daniel, J.L., and Kunapuli, S.P. 1998. Molecular basis for ADPinduced platelet activation. II. The P2Y1 receptor mediates ADP-induced intracellular calcium mobilization and shape change in platelets. J. Biol. Chem. 273:2030-2034

16. Hechler, B., Eckly, A., Ohlmann, P., and Cazenave, J.-P. 1998. The P2Y1 receptor, necessary but not sufficient to support full ADP-induced platelet aggregation, is not the target of the drug clopidogrel. $\mathrm{Br} . \mathrm{J}$. Haematol. 103:858-866.

17. Leon, C., et al. 2001. Key role of the P2Y1 receptor in tissue factorinduced thrombin-dependent acute thromboembolism: studies in P2Y1knockout mice and mice treated with a P2Y1 antagonist. Circulation. 103:718-723.

18. Trumel, C., et al. 1999. A key role of adenosine diphosphate in the irreversible platelet aggregation induced by the PAR1-activating peptide through the late activation of phosphoinositide 3-kinase. Blood. 94:4156-4165.

19. Martinson, E.A., Scheible, S., Marx-Grunwitz, A., and Presek, P. 1998. Secreted ADP plays a central role in thrombin-induced phospholipase D activation in human platelets. Thromb. Haemost. 80:976-981.

20. Cattaneo, M., and Gachet, C. 1999. ADP receptors and clinical bleeding disorders. Arterioscler. Thromb. Vasc. Biol. 19:2281-2285.

21. Geiger, J., et al. 1999. Specific impairment of human platelet P2Yac ADP receptor-mediated signaling by the antiplatelet drug clopidogrel. Arterioscler. Thromb. Vasc. Biol. 19:2007-2011.

22. Quinn, M.J., and Fitzgerald, D.J. 1999. Ticlopidine and clopidogrel. Circulation. 100:1667-1672.

23. Zhang, F.L., et al. 2001. ADP is the cognate ligand for the orphan G-protein coupled receptor SP1999. J. Biol. Chem. 276:8608-8615.

24. Bednar, B., Condra, C., Gould, R.J., and Connolly, T.M. 1995. Platelet aggregation monitored in a 96 well microplate reader is useful for evaluation of platelet agonists and antagonists. Thromb. Res. 77:453-463.

25. Fagura, M.S., et al. 1998. P2Y1-receptors in human platelets which are pharmacologically distinct from P2YADP-receptors. Br. J. Pharmacol. 124:157-164.

26. Baurand, A., et al. 2000. Desensitization of the platelet aggregation response to ADP: differential down-regulation of the P2Y1 and P2Ycyc receptors. Thromb. Haemost. 84:481-491.

27. Hollopeter, G., et al. 2001. Identification of the platelet ADP receptor targeted by antithrombotic drugs. Nature. 409:202-207.

28. Mahaut-Smith, M.P., Ennion, S.J., Rolf, M.G., and Evans, R.J. 2000. ADP is not an agonist at $\mathrm{P} 2 \mathrm{X} 1$ receptors: evidence for separate receptors stimulated by ATP and ADP on human platelets. Br. J. Pharmacol. 131:108-114.

29. Kahn, M.L., Nakanishi-Matsui, M., Shapiro, M.J., Ishihara, H., and Coughlin, S.R. 1999. Protease-activated receptors 1 and 4 mediate activation of human platelets by thrombin. J. Clin. Invest. 103:879-887. 